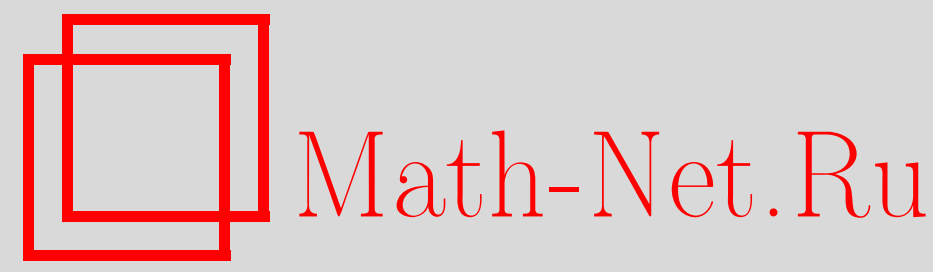

И. А. Тайманов, О вполне геодезических вложениях 7-мерных многообразий в 13мерные многообразия положительной секционной кривизны, Матем. сб., 1996, том 187, номер 12, 121-136

DOI: https://doi.org/10.4213/sm180

Использование Общероссийского математического портала Math-Net.Ru подразумевает, что вы прочитали и согласны с пользовательским соглашением

http://www.mathnet.ru/rus/agreement

Параметры загрузки:

IP: 54.224 .187 .69

26 апреля 2023 г., $12: 26: 50$ 
УДК 515.165.7

\author{
И. А. Тайманов
}

\title{
О вполне геодезических вложениях 7-мерных многообразий в 13-мерные многообразия положительной секционной кривизны
}

\begin{abstract}
В статье построены вполне геодезические вложения бесконечной серии 7-мерных многообразий в 13-мерные многообразия положительной секционной кривизны. Изучена топология этих вложений и обсуждена возможность построения новых многомерных примеров многообразий положительной кривизны.

Библиографйия: 12 названий.
\end{abstract}

\section{§1. Введение, основные результаты}

$\mathrm{K}$ настоящему времени известно довольно мало примеров замкнутых гладких многообразий, на которых существуют римановы метрики положительной секционной кривизны. Среди нечетномерных односвязных многообразий известные примеры исчерпываются сферами и 7- и 13-мерными многообразиями, построенными Берже [1], Алоффом и Уоллахом [2], Эшенбургом [3], [4] и Базайкиньгм [5]. На данных многообразиях конкретные примеры таких метрик были построены методами теории групп Ли.

Многообразия Берже и Алоффа-Уоллаха являются однородными, т.е. имеют вид $G / H$, где $G$ и $H \subset G$ - групшы Ли, а многообразия Эшенбурга и Базайкина - пространствами двойных частных, т.е. фактор-пространствами групп Ли $G$ по двусторонним изометрическим действиям некоторых иных групп Ли $H \subset G \times G$. Метод, примененный в [3], [5], впервые был предложен Громоллом и Майером при построении метрики неотрицательной секционной кривизны на экзотической семимерной сфере [6]. Заметим, что построенная ими метрика до сих пор остается единственным известным примером метрики такого типа, хотя попарно недиффеоморфные, но гомеоморфные многообразия положительной секционной кривизны были найдены Креком и Штольцем среди многообразий Алоффа-Уоллаха [7].

Взаимосвязь этих примеров до сих пор не исследовалась, хотя в результате прямых вычислений было обнаружено “странное" совпадение зашемленности метрик нормально однородного 13-мерного пространства Берже [1] и однородного 7-мерного пространства Алоффа-Уоллаха $N_{1,1,-1 / 2}$ (см. [2]). Защемленность метрик (отношение минимальной и максимальной секционных кривизн по двумерным направлениям) на них равна $\frac{16}{29 \cdot 37}$, как это было установлено Хайнтце для пространства Берже [8] и Хуангом для пространства Алоффа-Уоллаха [9]. Многообразия

Работа вьполнена при частичной поддержке Российского фонда фундаментальных исследований (грант № 96-01-01889). 
Эшенбурга и Базайкина являются неоднородными, что затрудняет вычисление защемленностей метрик на них. Среди них выделены такие серии многообразий $M_{j}$, что при $j \rightarrow \infty$ зашемленности метрик стремятся к $\frac{16}{29 \cdot 37}$ (см. [3], [10]).

В данной статье мы

- построим такую изометрическую инволюцию на 13-мерном пространстве Берже $B^{13}$, что одна из компонент множества неподвижных точек этой инволюции изометрична многообразию Алоффа-Уоллаха $N_{1,1,-1 / 2}$. Более того, максимум и минимум секционных кривизн пространства $B^{13}$ достигаются на плоскостях, касательных к данному вполне геодезическому многообразию $N_{1,1-1 / 2}$, что и объясняет совпадение их зашемленностей (теорема 1);

- построим на каждом 13-мерном пространстве Базайкина изометрическую инволюцию, для которой одна из компонент множества неподвижных точек будет совпадать с "отвечающим" этому 13-мерному пространству 7-мерным многообразием Эшенбурга (теорема 2);

- опишем топологию пространств $B^{13}$ и $N_{1,1,-1 / 2}$ и обсудим возможность продолжения вложения $N_{1,1,-1 / 2}$ в $B^{13}$ до башни вполне геодезических вложений (вопрос 2).

Мы избегаем во введении изложения технических деталей, которые однако указывают на большое родство 7-мерных пространств Алоффа-Уоллаха и Эшенбурга и 13-мерных пространств Берже и Базайкина, реализуемое, в частности, вполне геодезическими вложениями. Эти наблюдения и побудили нас сформулировать некоторые предположения (см. вопрос 1).

Автор благодарит У. Абреша и В. Майера, обративших его внимание на интересное совпадение зашемленностей для многообразий Берже и Алоффа-Уоллаха. Объяснение этого факта и явилось исходным пунктом этой работы. Автор благодарит рецензента за полезные замечания, способствовавшие улучшению текста.

Обозначения. Прежде, чем переходить к изложению результатов, вкратце остановимся на используемых нами обозначениях.

Мы будем отождествлять группы Ли $\mathrm{U}(n)$ с группами, образованными $(n \times n)$ матрицами $A$ такими, что

$$
A \cdot I_{n} \cdot A^{*}=I_{n},
$$

где $I_{n}$ - единичная $(n \times n)$-матрица и $A_{i j}^{*}=\bar{A}_{j i}$. Мы будем рассматривать группы $\mathrm{SU}(n)$ как подг руппы $\mathrm{U}(n)$, образованные матрицами с единичньм определителем. Под $\mathrm{Sp}(2)$ мы будем понимать подгруппу $\mathrm{SU}(4)$, состояшую из матрищ, удовлетворяющих условию

$$
A \cdot\left(\begin{array}{cc}
0 & I_{2} \\
-I_{2} & 0
\end{array}\right) \cdot A^{t}=\left(\begin{array}{cc}
0 & I_{2} \\
-I_{2} & 0
\end{array}\right),
$$

где $A_{i j}^{t}=A_{j i}$. Более того, мы будем также рассматривать $\operatorname{Sp}(2)$ как подгруппу $\mathrm{SU}(5)$, образованную матрицами вида

$$
\left(\begin{array}{cc}
A & 0 \\
0 & 1
\end{array}\right) \in \mathrm{SU}(5), \quad A \in \mathrm{Sp}(2) \subset \mathrm{SU}(4)
$$

Алгебры Ли этих групп мы тоже будем естественным образом отождествлять с матричными. 
Через $T_{\bar{p}}$ мы обозначим подгруппу $\mathrm{U}(5)$, образованную диагональными матрицами вида $\operatorname{diag}\left(z^{p_{1}}, z^{p_{2}}, z^{p_{3}}, z^{p_{4}}, z^{p_{5}}\right)$, где $|z|=1$ и $p_{1}, \ldots, p_{5}$ - неотрицательные целые числа. В случае, когда $\bar{p}=(1,1,1,1,-4)$ или $\bar{p}=(1,1,1,1,0)$, мы обозначим эти подгруппы через $T$ и $T_{0}$, соответственно.

\section{§2. Многообразия положительной секционной кривизны размерностей 7 и 13}

Прежде всего, напомним известные примеры 7- и 13-мерных односвязных многообразий, допускающих метрики положительной секционной кривизны.

1) 7-мерные многообразия.

1.1) 7-мерное пространство Берже [1].

Это пространство изометрично фактор-пространству $\mathrm{Sp}(2) / \mathrm{SU}(2)$, где группа $\operatorname{Sp}(2)$ оснащена стандартной двусторонне инвариантной метрикой, а вложение $\mathrm{SU}(2) \subset \mathrm{Sp}(2)$, в свою очередь, не является стандартным. Мы не будем обсуждать это пространство и будем рассматривать его как исключительное с точки зрения наших конструкций.

1.2) Пространства Алоффа-Уоллаха [2].

Обозначим через $T_{k, l}$ подгруппу $\mathrm{SU}(3)$, образованную диагональными матрицами вида $\operatorname{diag}\left(z^{k}, z^{l}, z^{-(k+l)}\right)$. Рассмотрим подгруппу $G_{1}=\mathrm{U}(2)$ группы $\mathrm{SU}(3)$, заданную следуюшим образом:

$$
G_{1}=\left(\begin{array}{cc}
A & 0 \\
0 & \operatorname{det} A^{-1}
\end{array}\right), \quad A \in \mathrm{U}(2)
$$

и обозначим через $g_{1}$ ее алгебру Ли. Через $f_{k, l}$ обозначим алгебру Ли группы $T_{k, l}$, вложенную в алгебру Ли групшы $\mathrm{SU}(3)$. Можно заметить, что $f_{k, l}$ порождена диагональными матрицами $\operatorname{diag}(2 \pi \sqrt{-1} k, 2 \pi \sqrt{-1} l,-2 \pi \sqrt{-1}(k+l))$. Обозначим через $\langle\cdot, \cdot\rangle_{0}$ двусторонне инвариантную метрику Киллинга на группе $\mathrm{SU}(3)$.

Определим однородную метрику на фактор-пространстве $N_{k, l}=\mathrm{SU}(3) / T_{k, l}$ следующей формулой:

$$
\langle x, y\rangle=(1+t)\left\langle x_{1}, y_{1}\right\rangle_{0}+\left\langle x_{2}, y_{2}\right\rangle_{0}
$$

где $x_{i}, y_{i} \in V_{i}$ и $f_{k, l}^{\perp}=V_{1} \oplus V_{2}$ - ортогональное разложение, $V_{1}=f_{k, l}^{\perp} \cap g_{1}$ и $V_{2}=g_{1}^{\perp}$. Полученное риманово многообразие обозначим через $N_{k, l, t}$.

Алофф и Уоллах показали, что если целые числа $k$ и $l$ одного и того же знака, то при $-1<t<0$ эти многообразия $N_{k, l, t}$ имеют положительную секционную кривизну.

1.3) Пространства Эшенбурга [3], [4].

Эти пространства обобщают пространства Алоффа-Уоллаха и имеют вид $T_{k_{1}, k_{2}, k_{3}} \backslash \mathrm{SU}(3) / T_{l_{1}, l_{2}, l_{3}}$, где $T_{k_{1}, k_{2}, k_{3}}=\operatorname{diag}\left(z^{k_{1}}, z^{k_{2}}, z^{k_{3}}\right), \quad|z|=1$. Для надлежашим образом выбранных троек целых чисел $\left(k_{1}, k_{2}, k_{3}\right)$ и $\left(l_{1}, l_{2}, l_{3}\right)$ мы получаем свободное действие на группе $\mathrm{SU}(3)$, фактор-пространство которого допускает метрику положительной секционной кривизны. Эти многообразия содержат первые известные примеры многообразий положительной секционной кривизны, которые негомеоморфны однородным. 
2) 13-мерные многообразия.

2.1) 13-мерное пространство Берже [1].

Это многообразие $B^{13}$ изометрично фактор-пространству $\mathrm{SU}(5) /(\operatorname{Sp}(2) \times T)$, где $\mathrm{SU}(5)$ наделено двусторонне инвариантной метрикой Киллинга.

2.2) Пространства Базайкина [5].

Эти пространства $M_{\bar{p}}^{13}$ имеют вид $T_{\bar{p}} \backslash \mathrm{U}(5) /\left(\mathrm{Sp}(2) \times T_{0}\right)$ и обобщают на 13 -мерньй случай пространства Эшенбурга. Если пространства Эшенбурга в некотором смысле можно рассматривать как "деформации" пространства Алоффа-Уоллаха, то пространства Базайкина, по аналогии, можно рассматривать как "деформации" пространства Берже $B^{13}$.

Если для всех подстановок $\tau \in S_{5}$ вьполняются следуюшие условия:

a) $p_{\tau(1)}+p_{\tau(2)}-p_{\tau(3)}-p_{\tau(4)}$ взаимно просто с $p_{\tau(5)}$;

б) $p_{\tau(1)}+p_{\tau(2)}+p_{\tau(3)}>p_{\tau(4)}+p_{\tau(5)}$;

в) $p_{\tau(1)}+p_{\tau(2)}+p_{\tau(3)}+p_{\tau(4)}>3 p_{\tau(5)}$

г) $3\left(p_{\tau(1)}+p_{\tau(2)}\right)>p_{\tau(3)}+p_{\tau(4)}+p_{\tau(5)}$,

то многообразие $M_{\bar{p}}^{13}=T_{\bar{p}} \backslash \mathrm{U}(5) /\left(\mathrm{Sp}(2) \times T_{0}\right)$ допускает метрику положительной секционной кривизны.

Мы также заметим, что эти метрики могут быть выбраны индуцированными из метрики на U(5) при факторизации, и при этом метрика на U(5) может быть выбрана левоинвариантной по отношению к действию всей группы U(5) и правоинвариантной по отношению к действию подгруппы $\mathrm{U}(4) \times \mathrm{U}(1)$, образованной блочньми матрицами с $4 \times 4$ - и $1 \times 1$-блоками. В дальнейшем мы будем считать, что эти многообразия оснашены такими метриками, построенными в [5].

\section{§3. Изометрическая инволюция} на 13-мерном пространстве Берже. Теорема 1

Перейдем теперь к нашей основной конструкции.

Положим

$$
S=\left(\begin{array}{ccccc}
-1 & 0 & 0 & 0 & 0 \\
0 & -1 & 0 & 0 & 0 \\
0 & 0 & 1 & 0 & 0 \\
0 & 0 & 0 & 1 & 0 \\
0 & 0 & 0 & 0 & 1
\end{array}\right)
$$

и определим следуюшее отображение:

$$
\sigma: \mathrm{U}(5) \rightarrow \mathrm{U}(5): A \rightarrow S \cdot A \cdot S^{-1} .
$$

Следующие утверждения очевидны.

ПРЕДЛОЖЕНИЕ 1. Справедливо равенство $\sigma(G)=G$ для $G=\mathrm{SU}(5)$, $\operatorname{Sp}(2), T_{\bar{p}}$.

ПРЕДЛОЖЕнИЕ 2. Отображение $\sigma^{2}$ - тождественно, т.е. $\sigma$ является инволюиией.

Матрица $S$ задает элемент групшы $\mathrm{SU}(5)$. При построении 13-мерного пространства Берже метрика на SU(5) берется двусторонне инвариантной [1], а при 
построении пространств Базайкина метрика на U(5) берется левоинвариантной по отношению к действию всей группы U(5) и правоинвариантной по отношению к действию подгрупшы $\mathrm{U}(4) \times \mathrm{U}(1)($ см. [5]). Так как $S \in \mathrm{SU}(5) \cap \mathrm{U}(4) \times \mathrm{U}(1) \subset \mathrm{U}(5)$, то инволюция $X \rightarrow S \cdot X \cdot S^{-1}$ является изометрией на группах $\mathrm{SU}(5)$ и $\mathrm{U}(5)$, оснашенных указанными выше метриками. Согласно предложению 1 эта инволюция сохраняет слои римановых субмерсий $\mathrm{SU}(5) \rightarrow B^{13}$ и $\mathrm{U}(5) \rightarrow M_{\bar{p}}^{13}$ и потому верно следуюшее предложение.

ПРЕДЛОЖЕНИЕ 3. Инволюиия $\sigma$ порождает изометрические инволюиии на пространствах $B^{13}$ и $M_{\bar{p}}^{13}$.

Рассмотрим сначала действие этой инволюции на пространстве Берже $B^{13}$.

ТЕОрема 1. Подмногообразие $W^{7} \subset B^{13}$, определенное как компонента множества неподвижных точек инволючии $\sigma: B^{13} \rightarrow B^{13}$, содержащая орбиту единицы группь $\mathrm{SU}(5)$, является вполне геодезическим подмногообразием пространства Берже $B^{13}$ и изометрично многообразию Алоффа-Уоллаха $N_{1,1,-1 / 2}$. При әтом секционная кривизна пространства Берэсе $B^{13}$ достигает своих минимальных и максимальных значений на плоскостях, касатель$\operatorname{Hblx} \kappa W^{7}$.

ДоКАЗАТЕЛЬСТво ТЕОРЕМЫ 1. 1) Топология множества неподвижных точек инволюции $\sigma: \mathrm{SU}(5) \rightarrow \mathrm{SU}(5)$ и определение $W^{7}$. Прямыми вычислениями легко показать, что если матрица $X \in \mathrm{U}(5)$ неподвижна под действием инволюции $\sigma$, то она имеет вид

$$
X=\left(\begin{array}{cc}
X_{1} & 0 \\
0 & X_{2}
\end{array}\right),
$$

где $X_{1} \in \mathrm{U}(2)$ и $X_{2} \in \mathrm{U}(3)$. Обозначим множество неподвижных точек инволюции $\sigma: \mathrm{SU}(5) \rightarrow \mathrm{SU}(5)$ через $F(\sigma)$ (оно выделяется условием $\operatorname{det} X_{1} \cdot \operatorname{det} X_{2}=1$ ).

Мы определим многообразие $W^{7}$ как образ $F(\sigma) \subset \mathrm{SU}(5)$ при факторизации

$$
F(\sigma) \subset \mathrm{SU}(5) \rightarrow \mathrm{SU}(5) /(\mathrm{Sp}(2) \times T)
$$

2) Действие $\sigma$ на касательном пространстве к $1 \in \mathrm{SU}(5)$. Мы отождествим касательное пространство к единице групшы $\mathrm{SU}(5)$ с алгеброй Ли $\mathrm{su}(5)$ и рассмотрим ортогональное разложение

$$
\mathrm{su}(5)=\mathbf{h} \oplus \mathbf{h}^{\perp}, \quad \mathbf{h}=\operatorname{sp}(2) \oplus \mathbb{R},
$$

где $\mathbf{h}$ - касательное пространство к подгруппе $\operatorname{Sp}(2) \times T$.

Действие инволюции $\sigma$ на $\mathrm{su}(5)$ обозначим тем же символом. Так как $\sigma: \mathrm{su}(5) \rightarrow \mathrm{su}(5)$ получается ограничением действия $\operatorname{Ad}_{S}: \mathrm{u}(5) \rightarrow \mathrm{u}(5)$ на подгруппу $\mathrm{su}(5) \subset \mathrm{u}(5)$, то оно является изометрией по отношению к форме Киллинга, что, впрочем, можно проверить и прямым вычислением.

Из предложения 1 следует, что

$$
\sigma(\mathbf{h})=\mathbf{h}
$$


Так как нас интересует структура множества неподвижных точек при действии на фактор-пространстве $B^{13}$, то мы ограничимся изучением действия инволюции на $\mathbf{h}^{\perp}$.

Выберем базис в $\mathbf{h}^{\perp}$ следующим образом. Обозначим через $E_{k l}$, где $1 \leqslant k, l \leqslant 5,(5 \times 5)$-матрицу $\left(\delta_{a k} \delta_{b l}\right)_{1 \leqslant a, b \leqslant 5}$. Введем матрицы $Q_{k l}=E_{k l}-E_{l k}$, $R_{k l}=\sqrt{-1}\left(E_{k l}+E_{l k}\right)$ и $P_{k}=\sqrt{-1}\left(E_{k k}-E_{55}\right)$.

Следующие матрицы задают ортонормированный базис в $\mathbf{h}^{\perp}$ (см. [8]):

$$
\begin{gathered}
M_{j}=\sqrt{2} Q_{j 5}, \quad M_{j+4}=\sqrt{2} R_{j 5}, \quad j=1,2,3,4 \\
M_{9}=Q_{12}-Q_{34}, \quad M_{10}=Q_{14}-Q_{23}, \\
M_{11}=R_{12}+R_{34}, \quad M_{12}=R_{14}-R_{23}, \quad M_{13}=P_{1}-P_{2}+P_{3}-P_{4} .
\end{gathered}
$$

Так как $\sigma$ действует на $\mathbf{h}^{\perp}$ изометрически и инволютивно, то существует разложение

$$
\mathbf{h}^{\perp}=V^{+} \oplus V^{-}
$$

такое, что

$$
\left.\sigma\right|_{V^{ \pm}}= \pm 1
$$

С помощью прямых вычислений, которые мы опускаем, можно показать, что

$$
\sigma\left(M_{k}\right)=\left\{\begin{aligned}
M_{k} & \text { при } k=3,4,7,8,9,11,13 \\
-M_{k} & \text { при } k=1,2,5,6,10,12
\end{aligned}\right.
$$

Отсюда следует, что $\operatorname{dim} V^{+}=7$ и $V^{+}$порождено векторами $M_{3}, M_{4}, M_{7}, M_{8}$, $M_{9}, M_{11}$ и $M_{13}$.

3) Многообразие $W^{7}$ как компонентамножества неподвижньгх точек инволюции $\sigma: B^{13} \rightarrow B^{13}$.

Лемма 1. Многообразие $W^{7}$ - однородное риманово многообразие ( с метрикой, индуцированной вложением $W^{7} \subset B^{13}$ ), вполне геодезично вложенное в $B^{13}$. Причем $W^{7}$ диффеоморфно многообразию Алоффа-Уоллаха $N_{1,1}$ и является компонентоймножества неподвижных точек инволюиии $\sigma: B^{13} \rightarrow B^{13}$.

ДоказАТЕЛЬСтво ЛЕммы 1. Прежде всего покажем, что $W^{7}$ является многообразием.

Подмногообразие $F(\sigma)$ состоит из матриц вида (2). Если умножение справа на матрицу из $g \in \mathrm{Sp}(2) \times T$ переводит матрицу вида (2) в матрицу такого же вида, то $g$ имеет вид:

$$
g=\left(\begin{array}{ccc}
A \cdot z & 0 & 0 \\
0 & \bar{A} \cdot z & 0 \\
0 & 0 & z^{-4}
\end{array}\right)
$$

где $A \in \mathrm{U}(2),|z|=1$.

Легко заметить, что любую матрицу из $F(\sigma)$ умножением справа на матрицу вида (3) можно привести к виду

$$
\left(\begin{array}{cc}
I_{2} & 0 \\
0 & A
\end{array}\right),
$$


где $A \in \mathrm{SU}(3)$.

Группа матриц вида (3) содержит подгруппу, образованную матрицами $\operatorname{diag}\left(1,1, z^{2}, z^{2}, z^{-4}\right),|z|=1$. Эта подгруппа действует на множестве матриц вида (4) правыми умножениями, и ее орбиты находятся во взаимно однозначном соответствии с точками пространства $W^{7}$.

Легко заметить, что $W^{7}$ диффеоморфно $N_{1,1}=\mathrm{SU}(3) / T_{1,1}$.

Так как метрика на SU(5) при построении $B^{13}$ выбирается двусторонне инвариантной, то подгруппа $\mathrm{SU}(3) \subset \mathrm{SU}(5)$, образованная матрицами вида (4), также оснащена двусторонне инвариантной метрикой. Она изометрически действует на $W^{7}$ умножениями слева, и мы заключаем, что риманово многообразие $W^{7}$ является однородным.

Нам осталось доказать, что $W^{7}$ является компонентой множества неподвижных точек инволюции $\sigma: B^{13} \rightarrow B^{13}$.

Из приведенных выше вычислений действия $\sigma$ на касательном пространстве к единице групшы $\mathrm{SU}(5)$ следует, что в окрестности единицы группы $\mathrm{SU}(5)$ (а точнее, в окрестности ее $(\mathrm{Sp}(2) \times T)$-орбиты) подмногообразие $W^{7}$ выделяется как множество неподвижных точек $\sigma$. Действие инволюции $\sigma: \mathrm{SU}(5) \rightarrow \mathrm{SU}(5)$ коммутирует с умножениями слева на матрищы вида (2) и любая точка $x \in W^{7}$ представима такой матрицей согласно определению пространства $W^{7}$. Отсюда заключаем, что $W^{7}$ выделяется условием $\sigma(x)=x \in B^{13}$ в окрестности любой своей точки, что и требовалось доказать.

Осталось заметить, что так как многообразие $W^{7}$ является компонентой множества неподвижных точек изометрической инволюции, то оно является вполне геодезическим подмногообразием. Лемма 1 доказана.

4) Однородная метрика на $W^{7}$.

Лемма 2. Риманово многообразие $W^{7}$ изометрично многообразию Алоффа-Уоллаха $N_{1,1,-1 / 2}$.

ДокАЗАТЕЛЬСтво ЛЕммы 2. Прежде всего заметим, что

$$
M_{9}=-2 Q_{34}+v_{1}, \quad M_{11}=2 R_{34}+v_{2}, \quad M_{13}=2\left(P_{3}-P_{4}\right)+v_{3},
$$

где

$$
\begin{aligned}
& v_{1}=\left(\begin{array}{ccccc}
0 & 1 & 0 & 0 & 0 \\
-1 & 0 & 0 & 0 & 0 \\
0 & 0 & 0 & 1 & 0 \\
0 & 0 & -1 & 0 & 0 \\
0 & 0 & 0 & 0 & 0
\end{array}\right), \\
& v_{2}=\left(\begin{array}{ccccc}
0 & \sqrt{-1} & 0 & 0 & 0 \\
\sqrt{-1} & 0 & 0 & 0 & 0 \\
0 & 0 & 0 & -\sqrt{-1} & 0 \\
0 & 0 & -\sqrt{-1} & 0 & 0 \\
0 & 0 & 0 & 0 & 0
\end{array}\right), \\
& v_{3}=\left(\begin{array}{ccccc}
\sqrt{-1} & 0 & 0 & 0 & 0 \\
0 & -\sqrt{-1} & 0 & 0 & 0 \\
0 & 0 & -\sqrt{-1} & 0 & 0 \\
0 & 0 & 0 & \sqrt{-1} & 0 \\
0 & 0 & 0 & 0 & 0
\end{array}\right) .
\end{aligned}
$$


Так как $v_{1}, v_{2}, v_{3} \in \mathbf{h} \subset \mathrm{su}(5)$ и отображение $\mathrm{SU}(5) \rightarrow B^{13}$ является римановой субмерсией, отсюда следует, что $\left(M_{3}, M_{4}, M_{7}, M_{8}, 2 Q_{34}, 2 R_{34}, 2\left(P_{3}-P_{4}\right)\right)$ образуют ортонормированный базис в касательном пространстве $\kappa W^{7}$ в точке, отвечающей $(\mathrm{Sp}(2) \times T)$-орбите единицы группь $\mathrm{SU}(5)$.

В то же время известно, что $\left(M_{3}, M_{4}, M_{7}, M_{8}, \sqrt{2} Q_{34}, \sqrt{2} R_{34}, \sqrt{2}\left(P_{3}-P_{4}\right)\right)$ образуют ортонормированный (с точностью до умножения на постоянную) базис в подалгебре $V_{1}$ (см. (1)) для $k=l=1$.

Отсюда мы заключаем, что вложение $W^{7} \rightarrow B^{13}$ индуцирует на $W^{7}$ метрику Алоффа-Уоллаха (1) с $t=-\frac{1}{2}$. Лемма 2 доказана.

5) Кривизна пространства $W^{7}$. Зашемленность метрики. Мы воспользуемся формулами, полученньми в [8]. Так как риманово многообразие $W^{7}$ однородно, то достаточно посчитать кривизны в одной его точке. Мы рассмотрим точку, отвечаюшую $(\mathrm{Sp}(2) \times T)$-орбите единицы группы $\mathrm{SU}(5)$.

C помошью формул из [8] мы находим кривизну в направлении, заданном плоскостью, натянутой на касательные векторы $M_{3}$ и $M_{7}$ :

$$
K\left(M_{3}, M_{7}\right)=\frac{29}{4} .
$$

Более того, в [8] доказано, что $\frac{29}{4}$ является наибольшим возможным значением секционной кривизны пространства $B^{13}$.

Рассмотрим матрицу

$$
F=\frac{1}{\sqrt{2}}\left(\begin{array}{ccccc}
1 & 0 & -\sqrt{-1} & 0 & 0 \\
0 & \sqrt{-1} & 0 & 1 & 0 \\
-\sqrt{-1} & 0 & 1 & 0 & 0 \\
0 & -1 & 0 & -\sqrt{-1} & 0 \\
0 & 0 & 0 & 0 & \sqrt{2}
\end{array}\right)
$$

Так как $F \in \mathrm{Sp}(2) \subset \mathrm{SU}(5)$, то действие $\xi: X \rightarrow F \cdot X \cdot F^{-1}$ порождает изометрию пространства $B^{13}$.

Пусть

$$
\begin{aligned}
& X=\sqrt{\frac{12}{37}}\left(M_{7}+M_{8}\right)+\sqrt{\frac{13}{37}} M_{11}, \\
& Y=\sqrt{\frac{12}{37}}\left(M_{3}-M_{4}\right)+\sqrt{\frac{13}{37}} M_{9} .
\end{aligned}
$$

Легко заметить, что $X, Y \in V^{+} \subset \mathbf{h}^{\perp}$ и потому задают касательные векторы к пространству $W^{7}$.

Чтобы применить формулы из [8], перейдем к другой паре векторов

$$
\begin{aligned}
& X_{0}=\xi(X)=\sqrt{\frac{12}{37}}\left(\frac{M_{1}+M_{7}}{\sqrt{2}}+\frac{M_{4}+M_{6}}{\sqrt{2}}\right)+\sqrt{\frac{13}{37}} M_{9}, \\
& Y_{0}=\xi(Y)=\sqrt{\frac{12}{37}}\left(\frac{M_{3}-M_{5}}{\sqrt{2}}-\frac{M_{2}-M_{8}}{\sqrt{2}}\right)+\sqrt{\frac{13}{37}} M_{10}
\end{aligned}
$$


к которым и применим лемму 2 работы [8]. Прямыми вычислениями получаем, что кривизна $B^{13}$ в направлении, заданном касательньми векторами $X$ и $Y$, равна $\frac{4}{37}$ :

$$
K(X, Y)=K\left(X_{0}, Y_{0}\right)=\frac{4}{37}
$$

В [8] доказано, что это значение является минимальньм среди возможных значений секционных кривизн пространства $B^{13}$.

Отсюданемедленно следует, что зашемленностиметрик на $B^{13}$ и $W^{7}=N_{1,1,-1 / 2}$ совпадают и равны $\frac{16}{29 \cdot 37}=\frac{16}{1073}$.

Теорема 1 доказана.

\section{§4. Изометрические инволюции на пространствах Базайкина. Теорема 2}

С помощью аналогичных рассуждений можно доказать следующий аналог теоремы 1.

ТЕОРема 2. Компонента множества неподвижных точек инволюиии $\sigma: M_{\bar{p}}^{13} \rightarrow M_{\bar{p}}^{13}$, содерәсащая $T_{p} \times\left(\mathrm{Sp}(2) \times T_{0}\right)$-орбиту единиць группьи $\mathrm{U}(5)$, является вполне геодезическим подмногообразием $W_{\bar{p}}^{7}$, изометричным про-

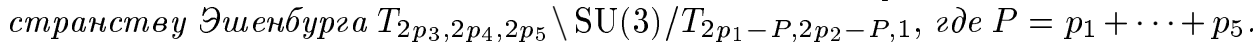

ДоКАЗАТЕЛЬСТво ТЕОРЕМЫ 2. Как и в случае действия инволюции на пространстве Берже, в окрестности единицы группы U(5) компонента множества неподвижных точек инволюции задается элементами вида (2). Так как эти элементы образуют подмногообразие $F(\sigma)$, то искомая компонента $W_{\bar{p}}^{7}$ состоит из $\mathrm{U}(1) \times(\mathrm{U}(4) \times \mathrm{U}(1))$-орбит точек подмногообразия $F(\sigma)$.

Рассуждения, аналогичные приведенным при доказательстве леммы 1 , показывают, что

$$
\begin{gathered}
W_{\bar{p}}=\left(\begin{array}{ccc}
z^{p_{3}} & 0 & 0 \\
0 & z^{p_{4}} & 0 \\
0 & 0 & z^{p_{5}}
\end{array}\right) \backslash \mathrm{U}(3) /\left(\begin{array}{ccc}
w \cdot z^{p_{1}} & 0 & 0 \\
0 & w \cdot z^{p_{2}} & 0 \\
0 & 0 & 1
\end{array}\right) \\
|z|=|w|=1 .
\end{gathered}
$$

Так как $W_{\bar{p}}^{7}$ являются компонентами множеств неподвижных точек изометрических инволюций (согласно предложению 3), то они являются вполне геодезическими подмногообразиями $M_{\bar{p}}^{13}$ и, в частности, имеют положительную секционную кривизну.

Так как пространства $M_{\bar{p}}^{13}$ неоднородны (за исключением случая, когда $\left.p_{1}=\cdots=p_{5}=1\right)$, то задача сравнения защемленностей метрик на $W_{\bar{p}}^{7}$ и $M_{\bar{p}}{ }^{3}$ не сводится к локальньм вычислениям в одной точке, как это было сделано при доказательстве теоремы 1.

Сравним пространства $W_{\bar{p}}^{7}$ с пространствами Алофффа-Уоллаха и Эшенбурга. 
Так как пятерка чисел $\bar{p}=\left(p_{1}, p_{2}, p_{3}, p_{4}, p_{5}\right)$ удовлетворяет условиям а)-г), входящим в определение пространств $M_{\bar{p}}^{13}$ (см. $\S 2$, п. 2.2), то она, в частности, удовлетворяет условию а). Отсюда немедленно следует, что сумма $P=p_{1}+\cdots+p_{5}$ нечетна.

Заменим $z=\exp (\varphi)$ на $u=\exp (\varphi / 2)$, а $w$ на $v \cdot u^{-P}$. В этих обозначениях пространство $W_{\bar{p}}^{7}$ имеет вид:

$$
W_{\bar{p}}^{7}=\left(\begin{array}{ccc}
u^{2 p_{3}} & 0 & 0 \\
0 & u^{2 p_{4}} & 0 \\
0 & 0 & u^{2 p_{5}}
\end{array}\right) \backslash \mathrm{U}(3) /\left(\begin{array}{ccc}
v \cdot u^{2 p_{1}-P} & 0 & 0 \\
0 & v \cdot u^{2 p_{2}-P} & 0 \\
0 & 0 & 1
\end{array}\right),
$$

где $|u|=|v|=1$. Действие двумерного тора $T^{2}(u, v)=T(u) \times T(v)$, где $T(u)=$ $\{|u|=1\}, T(v)=\{|v|=1\}$, на $\mathrm{U}(3)$ не будет свободным, а будет иметь ядро $\mathbb{Z}_{2}=((1,1),(-1,-1))$, т.е. свободным будет действие тора $T^{2}(u, v) / \mathbb{Z}_{2}$.

Фактор-пространство $\Gamma=\mathrm{U}(3) / T(v)$ диффеоморфно фактор-пространству $\mathrm{SU}(3)$ по правому действию группы $\mathbb{Z}_{2}$, порожденному элементом

$$
\gamma=\left(\begin{array}{cc}
-I_{2} & 0 \\
0 & 1
\end{array}\right) \in \mathrm{SU}(3)
$$

Так как $P$ нечетно, то действие элемента $\gamma$ совпадает с действием элемента $\widetilde{\gamma} \in T(u)$, отвечающего значению $u=-1$. С этим совпадением и связано наличие ядра у действия $T^{2}(u, v)$ на $\mathrm{U}(3)$.

Теперь легко заметить, что фактор-пространство Г/T(u) диффеоморфно фактор-пространству групшы $\mathrm{SU}(3)$ по двустороннему действию $T(u)$, задаваемому формулой

$$
X \rightarrow \operatorname{diag}\left(u^{2 p_{3}}, u^{2 p_{4}}, u^{2 p_{5}}\right) \cdot X \cdot \operatorname{diag}\left(u^{2 p_{1}-P}, u^{2 p_{2}-P}, 1\right) .
$$

Из того, что пятерка чисел $\left(p_{1}, \ldots, p_{5}\right)$ удовлетворяет условию а), и из нечетности $P$ следует, что это действие свободно.

Таким образом, мы показали, что

$$
W_{\bar{p}}^{7}=T_{2 p_{3}, 2 p_{4}, 2 p_{5}} \backslash \mathrm{SU}(3) / T_{2 p_{1}-P, 2 p_{2}-P, 1},
$$

где $P=p_{1}+\cdots+p_{5}$.

Здесь следует напомнить определение метрик на пространствах Эшенбурга и Базайкина.

При построении пространств Эшенбурга метрика на групе U(3) имеет вид:

$$
\langle x, y\rangle=t\left\langle x_{1}, y_{1}\right\rangle_{0}+\left\langle x_{2}, y_{2}\right\rangle_{0},
$$

где $x_{i}, y_{i} \in V_{i}, \mathrm{u}(3)=V_{1} \oplus V_{2}$ - ортогональное разложение, $V_{1}$ - касательная алгебра к диагонально вложенной подгруппе $K=\mathrm{U}(2) \times \mathrm{U}(1),\langle\cdot, \cdot\rangle_{0}-$ двусторонне-инвариантная метрика Киллинга, $t \in \mathbb{R}$. При построении пространств Базайкина метрика на U(5) выбирается аналогичным образом, только подгруппа $K$ имеет вид $\mathrm{U}(4) \times \mathrm{U}(1)$ (вложение также диагонально). 
В [4] и [5] было показано, что при $t=\frac{1}{2}$ метрики полученные на пространствах двойных частных имеют положительную секционную кривизну. Очевидно, что параметр $t$ может варьироваться, однако пределы вариаций, при которых положительность секционной кривизны сохраняется, не оценивались.

Как и при доказательстве леммы 2 , из (5) выводится, что если метрика на $M_{\bar{p}}^{13}$ отвечает значению $t=t_{0}$, то метрика на $W_{\bar{p}}^{7}$ отвечает значению $t=t_{0} / 2$.

Теорема 2 доказана.

В простейшем случае, когда $p_{1}=1, p_{2}=\cdots=p_{5}=q$, где $q>1$, мы получаем пространство диффеоморфное (но не изометричное) однородному многообразию $N_{2 q-1,1}$.

Следуюший вопрос представляется интересным.

Вопрос 1. Существует ли соответствие между 7-мерными пространствами Алоффа-Уоллаха и Эшенбурга и 13-мерными пространствами Базайкина, которое реализуется вполне геодезическими вложсениями?

Если такое соответствие существует лишь для каких-то семейств многообразий, то как устроены эти семейства?

Если такое соответствие существует, то реализуется ли оно вполне геодезическими вложсениями, сохраняющими защемленности метрик?

§5. Топология многообразий Алоффа-Уоллаха $N_{1,1}$ и Берже $B^{13}$. Башни вполне геодезических вложений

Рассмотрим топологическое строение пространств $W^{7}=N_{1,1,-1 / 2}$ и $B^{13}$.

Пусть

$$
\widehat{G}(\approx \mathrm{SU}(2))=\left(\begin{array}{cc}
A & 0 \\
0 & 1
\end{array}\right) \subset \mathrm{SU}(3), \quad A \in \mathrm{SU}(2)
$$

Можно заметить, что $\mathrm{SU}(3) / T_{1,1}=W^{7}$ и $\mathrm{SU}(3) / \widehat{G}=S^{5}$. Группа $T_{1,1}$ действует на $S^{5}=\left\{z_{1}^{2}+z_{2}^{2}+z_{3}^{2}=1 \mid z_{i} \in \mathbb{C}\right\}$ умножениями:

$$
\left(z_{1}, z_{2}, z_{3}\right) \rightarrow\left(\lambda^{-2} z_{1}, \lambda^{-2} z_{2}, \lambda^{-2} z_{3}\right)
$$

где $\operatorname{diag}\left(\lambda, \lambda, \lambda^{-2}\right) \in T_{1,1}$. Более того, действия $T_{1,1}$ и $\widehat{G}$ на $\mathrm{SU}(3)$ коммутируют.

Рассмотрим расслоение

$$
\mathrm{SU}(3) \rightarrow \mathbb{C P}^{2}
$$

Его слой диффеоморфен группе U(2), которую можно представить следующим образом. Пусть

$$
\widehat{Q}=\mathrm{SU}(2) \times(\mathbb{R} / 2 \pi \mathbb{Z})
$$

Обозначим через $\widehat{Q}_{1}$ фактор-пространство $\widehat{Q} / \mathbb{Z}_{2}$, где $\mathbb{Z}_{2}$-действие имеет вид:

$$
(X, t) \rightarrow(-X, t+\pi), \quad X \in \mathrm{SU}(2)
$$


Это фактор-пространство диффеоморфно слою расслоения (6). Оно расслаивается над $S^{1}$ обычным образом:

$$
(X, t) \rightarrow t \in S^{1}=\mathbb{R} / \pi \mathbb{Z} .
$$

В этих обозначениях действие $T_{1,1}$ на слоях расслоения (6) имеет вид:

$$
\begin{gathered}
(X, t) \rightarrow(\exp (\sqrt{-1} \pi \varphi) \cdot X, t+\varphi) \\
\operatorname{diag}(\exp (\sqrt{-1} \pi \varphi), \exp (\sqrt{-1} \pi \varphi), \exp (-2 \sqrt{-1} \pi \varphi)) \in T_{1,1} .
\end{gathered}
$$

Отсюда выводим, что

1) $S^{5} / T_{1,1}=\mathrm{SU}(3) / \widehat{G} \times T_{1,1}=\mathbb{C P}^{2}$;

2) эти действия порождают расслоение

$$
W^{7}=\mathrm{SU}(3) / T_{1,1} \stackrel{\mathbb{R P}^{3}}{\longrightarrow} \mathbb{C P}^{2}
$$

3) из вычисления групп когомологий пространства $W^{7}$ (см. [3]) следует, что трансгрессия $d_{4}$ в спектральной последовательности расслоения (7) задается следующей формулой:

$$
d_{4}: E_{4}^{0,3}=\mathbb{Z} \stackrel{\times 3}{\longrightarrow} E_{4}^{4,0}=\mathbb{Z}
$$

и

$$
H^{4}\left(W^{7}\right)=\mathbb{Z}_{3}
$$

Пусть

$$
\widetilde{G}(\approx \mathrm{SU}(4))=\left(\begin{array}{ll}
A & 0 \\
0 & 1
\end{array}\right) \subset \mathrm{SU}(5), \quad A \in \mathrm{SU}(4) .
$$

Можно заметить, что $\mathrm{SU}(5) / \mathrm{Sp}(2) \times T=B^{13}$ и $\mathrm{SU}(5) / \widetilde{G}=S^{9}$. Группа $T$ действует на $S^{9}=\left\{z_{1}^{2}+\cdots+z_{5}^{2}=1 \mid z_{i} \in \mathbb{C}\right\}$ умножениями:

$$
\left(z_{1}, \ldots, z_{5}\right) \rightarrow\left(\lambda^{-4} z_{1}, \ldots, \lambda^{-4} z_{5}\right)
$$

где $\operatorname{diag}\left(\lambda, \lambda, \lambda, \lambda, \lambda^{-4}\right) \in T$. Более того, действия $T$ и $\widetilde{G}$ на $\mathrm{SU}(5)$ коммутируют.

Рассмотрим расслоение

$$
\mathrm{SU}(5) / \mathrm{Sp}(2) \rightarrow \mathbb{C P}^{4}
$$

Пусть

$$
\widetilde{Q}=\operatorname{SU}(4) / \operatorname{Sp}(2) \times(\mathbb{R} / \pi \mathbb{Z}) .
$$

Обозначим через $\widetilde{Q}_{1}$ фактор-пространство $\widetilde{Q} / \mathbb{Z}_{2}$, где $\mathbb{Z}_{2}$-действие имеет вид:

$$
(X, t) \rightarrow\left(\sqrt{-1} X, t+\frac{\pi}{2}\right), \quad X \in \mathrm{SU}(4) / \mathrm{Sp}(2) .
$$


Это фактор-пространство диффеоморфно слою расслоения (10). Оно расслаивается над $S^{1}$ обычным образом:

$$
(X, t) \rightarrow t \in S^{1}=\mathbb{R} / \frac{\pi}{2} \mathbb{Z} .
$$

В этих обозначениях действие $T$ на слоях расслоения (10) имеет вид:

$$
\begin{gathered}
(X, t) \rightarrow(\exp (\sqrt{-1} \pi \varphi) \cdot X, t+\varphi), \\
\operatorname{diag}(\exp (\sqrt{-1} \pi \varphi), \ldots, \exp (\sqrt{-1} \pi \varphi), \exp (-4 \sqrt{-1} \pi \varphi)) \in T .
\end{gathered}
$$

Отсюда выводим, что

1) $S^{5} / T=\mathrm{SU}(5) / \widetilde{G} \times T=\mathbb{C P}^{4}$;

2) эти действия порождают расслоение

$$
B^{13} \stackrel{\mathbb{R P}^{5}}{\longrightarrow} \mathbb{C P}^{4}
$$

3) из вычислений групп когомологий пространства $B^{13}$ (см. [5]) следует, что трансгрессия $d_{6}$ в спектральной последовательности расслоения (11) задается следующей формулой

$$
d_{6}: E_{6}^{0,5}=\mathbb{Z} \stackrel{\times 5}{\longrightarrow} E_{6}^{6,0}=\mathbb{Z}
$$

и

$$
H^{6}\left(B^{13}\right)=\mathbb{Z}_{5}
$$

Подобие формул (6)-(9) для $W^{7}$ и формул (10)-(13) для $B^{13}$ и теорема 1 дают нам основание поставить следующий вопрос.

Вопрос 2. Верно ли, что для каждого положительного целого числа $k$ существует пространство $\Gamma_{k}$ такое, что

1) существует расслоение

$$
\Gamma_{k} \stackrel{\mathbb{R P}^{2 k+1}}{\longrightarrow} \mathbb{C P}^{2 k}
$$

2) трансгрессия $d_{2 k+2}$ в спектральной последовательности расслоения (14) имеет вид:

$$
d_{2 k+2}: E_{2 k+2}^{0,2 k+1}=\mathbb{Z} \stackrel{\times(2 k+1)}{\longrightarrow} H^{2 k+2}\left(\mathbb{C P}^{2 k}\right)
$$

$u$

$$
H^{2 k+2}\left(\Gamma_{k}\right)=\mathbb{Z}_{2 k+1}
$$

3) многообразие $\Gamma_{k}$ имеет положсительную секиионную кривизну;

4) $\Gamma_{1}=W^{7} u \Gamma_{2}=B^{13}$ ?

Можно предложить более строгую гипотезу, добавив следуюшее:

5) пространства $\Gamma_{k}$ образуют башню

$$
\Gamma_{1} \rightarrow \Gamma_{2} \rightarrow \cdots \rightarrow \Gamma_{n} \rightarrow \Gamma_{n+1} \rightarrow \cdots
$$

вполне геодезических вложений, сохраняющих защемленности метрик, и, тем самым, защемленности пространств $\Gamma_{k}$ равны $\frac{16}{29 \cdot 37}$.

Если такая башня сушествует, то можно ожидать, что ее свойства похожи на свойства $\mathbb{C P}^{n}$ - и $\mathbb{H}^{n}$-башен. 


\section{§6. Заключительные замечания}

6.1. Рассмотрим вкратце топологию простейших пространств вида $W_{\bar{p}} \frac{7}{13}$. А именно, обозначим через $W_{p}^{7}$ и $M_{p}^{13}$ пространства такого вида, отвечающие $\bar{p}=(p, p, p, p, 1)$.

Так как левостороннее действие сохраняет орбитыправостороннего действия, то групша $\widetilde{T}_{p}=\operatorname{diag}\left(z^{p}, z^{p}, z\right)$ действует на пространстве $\mathrm{U}(3) /(\mathrm{SU}(2) \times \operatorname{diag}(w, w, 1))$ $=S^{5}$, где $|z|=|w|=1$, а группа $T_{p}=\operatorname{diag}\left(z^{p}, z^{p}, z^{p}, z^{p}, z\right)$ действует на пространстве $\mathrm{U}(5) /\left(\mathrm{SU}(4) \times T_{0}\right)=S^{9}$.

Эти действия не являются свободными.

Можно заметить, что элементы $\operatorname{diag}\left(z^{p}, z^{p}, z\right)$ при $z^{p}=1$ имеют нетривиальное множество неподвижных точек, а другие элементы $\widetilde{T}_{p}$ действуют свободно. Эти множества неподвижных точек одни и те же для всех корней $p$-й степени из единипы и диффеоморфны 3 -мерной экваториальной сфере в $S^{5}$. Рассмотрим $\mathbb{Z}_{p}$-действие на $S^{5}$, задаваемое действием подгрупшы $\widetilde{T}_{p}$, образованной корнями $p$-й степени из единицы $\left(z^{p}=1\right)$. Фактор-пространство $\mathrm{U}(3) /(\mathrm{SU}(2) \times \operatorname{diag}(w, w, 1))$ относительно этого $\mathbb{Z}_{p}$-действия опять диффеоморфно $S^{5}$ (это легко представить, рассмотрев $S^{5}$ как циклическое $p$-листное накрытие $S^{5}$, разветвленное по экваториальной 3 -мерной сфере). Фактор-группа $\widetilde{T}_{p} / \mathbb{Z}_{p}$ действует на этом фактор-пространстве свободно, и для каждого $p$ мы получаем отображение

$$
W_{p}^{7} \rightarrow \operatorname{diag}\left(z^{p}, z^{p}, z\right) \backslash \mathrm{U}(3) /(\mathrm{SU}(2) \times \operatorname{diag}(w, w, 1))=\mathbb{C P}^{2} .
$$

Почти очевидно, что это отображение задает $\mathbb{R} \mathbb{P}^{3}$-расслоение, но строгое доказательство требует дополнительной работы.

Недавно Я.В. Базайкин, отвечая на наш вопрос, вычислил порядок группы $H^{4}\left(W_{p}^{7}\right)$, которьй, как оказалось, равен $r_{p}=(4 p-1)$.

Ситуация в размерности 13 совершенно аналогична 7-мерной, и для каждого $p$ мы получаем отображение

$$
M_{p}^{13} \rightarrow T_{p}^{1} \backslash \mathrm{U}(5) /\left(\mathrm{SU}(4) \times T_{0}\right)=\mathbb{C P}^{4}
$$

Это отображение тоже должно являться $\mathbb{R} \mathbb{P}^{5}$-расслоением.

В [5] было показано, что порядок групшы $H^{6}\left(M_{p}^{13}\right)$ равен $s_{p}=\left(8 p^{2}-4 p+1\right)$.

Можно заметить очень интересное соотношение

$$
s_{p}=\frac{r_{p}^{2}+1}{2}
$$

которое для $p=1$ совпадает с (9) и (13).

Можно также задаться вопросом о том, продолжаемы ли эти вложения $W_{p}^{7} \rightarrow M_{p}^{13}$ до башен вполне геодезических вложений. 
6.2. Когда мы обсуждали результаты этой работы с К. Грове, он задал вопрос о сушествовании таких вложений для четномерных пространств Уоллаха [11]. Сушествование топологических вложений для них очевидно. Мы можем указать простейший пример инволюции

$$
\widehat{S}=\left(\begin{array}{cc}
\sqrt{-1} I_{3} & 0 \\
0 & -\sqrt{-1} I_{3}
\end{array}\right),
$$

которая порождает инволюцию 12-мерного пространства Уоллаха $\operatorname{Sp}(3) /(\operatorname{Sp}(1) \times$ $\mathrm{Sp}(1) \times \mathrm{Sp}(1))$. Компонента множества неподвижных точек, содержашая орбиту единицы группы $\mathrm{Sp}(3)$, диффеоморфна 6-мерному пространству Уоллаха $\mathrm{SU}(3) / T^{2}$, где $T^{2}$ - максимальный тор групшы $\mathrm{SU}(3)$. Тем самым мы получаем вполне геодезическое вложение $\mathrm{SU}(3) / T^{2} \rightarrow \mathrm{Sp}(3) /(\mathrm{Sp}(1) \times \mathrm{Sp}(1) \times \mathrm{Sp}(1))$.

В [12] было показано, что максимальные защемленности однородных метрик на всех четномерных пространствах Уоллаха совпадают и равны $\frac{1}{64}$. Поэтому следует ожидать, что это вложение сохраняет защемленность римановой метрики и что для другой пары пространств Уоллаха $\left(F_{4} / \mathrm{Spin}(8), \operatorname{Sp}(3) /(\mathrm{Sp}(1) \times \mathrm{Sp}(1) \times \mathrm{Sp}(1))\right)$ такое вложение тоже сушествует.

Однако, само сушествование этих пространств обусловлено существованием конечномерных алгебр с делением над полем $\mathbb{R}$, которые согласно теореме Адамса существуют лишь в размерностях $1,2,4$ и 8 . Поэтому не следует ожидать, что башшя четномерных пространств Уоллаха может быть продолжена.

6.3. Эта статья является переработанньм и расширенным вариантом препринта автора "A remark on positively curved manifolds of dimensions 7 and 13" (cм. dg-ga/9511017).

\section{Список литературы}

1. Berger $M$. Les varietes homogenes normales simplement connexes a courbure strictment positive // Ann. Scuola Norm. Sup. Pisa. 1961. V. 15. P. 179-216.

2. Aloff S., Wallach N.R. An infinite family of distinct 7-manifolds admitting positively curved Riemannian structures // Bull. Amer. Math. Soc. 1975. V. 81. P. 93-97.

3. Eschenburg J.-H. New examples of manifolds with strictly positive curvature // Invent. Math. 1982. V. 66. P. 469-480.

4. Eschenburg J.-H. Inhomogeneous spaces of positive curvature // Differential Geom. Appl. 1992. V. 2. P. 123-132.

5. Базайкин Я.В. Об одном семействе 13-мерных замкнутых римановых многообразий положительной кривизны // Сиб. матем. журн. 1996. Т. 37. С. 1219-1237.

6. Gromoll D., Meyer W. An exotic sphere with nonnegative sectional curvature // Ann. of Math. 1974. V. 100. P. 401-406.

7. Kreck M., Stolz S. Some nondiffeomorphic homeomorphic homogeneous 7-manifolds with positive sectional curvature // J. Differential Geom. 1991. V. 33. P. 465-486.

8. Heintze E. The curvature of $\mathrm{SU}(5) /\left(\mathrm{Sp}(2) \times S^{1}\right) / /$ Invent. Math. 1971. V. 13. P. 205-212.

9. Huang H. M. Some remarks on the pinching problems // Bull. Inst. Math. Acad. Sinica. 1981. V. 9. P. 321-340. 
10. Abresch U., Meyer W.T. Injectivity radius estimates and sphere theorems // Proc. of MSRI (to appear).

11. Wallach $N$. Compact homogeneous Riemannian manifolds with strictly positive curvature // Ann. of Math. 1972. V. 96. P. 277-295.

12. Валиев Ф. М. Точные оценки секционных кривизн однородных римановых метрик на пространствах Уоллача // Сиб. матем. журн. 1979. Т. 20. С. 248-262.

Институт математики СО РАН

Поступила в редакцию

31.01 .1996 